\title{
Binding of Biologically Relevant Divalent Cations to Aqueous Carboxylates: Molecular Simulations Guided by Raman Spectroscopy
}

\author{
Denilson Mendes de Oliveira ${ }^{1}$, Samual R. Zukowski ${ }^{1}$, Vladimir Palivec ${ }^{2}$, Jérôme Hénin ${ }^{3,4}$, \\ Hector Martinez Seara ${ }^{2}$, Dor Ben Amotz ${ }^{1 *}$, Pavel Jungwirth ${ }^{2 *}$, Elise Duboué-Dijon ${ }^{2,3,4^{*}}$ \\ ${ }^{1}$ Department of Chemistry, Purdue University, West Lafayette, Indiana 47907 \\ ${ }^{2}$ Institute of Organic Chemistry and Biochemistry, Academy of Sciences of the Czech Republic, \\ Flemingovo nám. 2, 16610 Prague 6, Czech Republic \\ ${ }^{3}$ CNRS, Université de Paris, UPR 9080, Laboratoire de Biochimie Théorique, 13 rue Pierre et \\ Marie Curie, 75005, Paris, France \\ ${ }^{4}$ Institut de Biologie Physico-Chimique-Fondation Edmond de Rothschild, PSL Research \\ University, Paris, France \\ *email: pavel.jungwirth@uochb.cas.cz; bendor@purdue.edu;duboue-dijon@ibpc.fr
}

\begin{abstract}
In spite of the biological importance of the binding of $\mathrm{Zn}^{2+}, \mathrm{Ca}^{2+}$, and $\mathrm{Mg}^{2+}$ to carboxylate anions, previous experimental and computational studies have reached conflicting conclusions regarding the corresponding binding affinities. Here, we report the first use of Raman multivariate curve resolution (Raman-MCR) vibrational spectroscopy to obtain self-consistent free and bound metal acetate spectra and one-to-one binding constants, without the need to invoke any a priori assumptions regarding the shapes of the corresponding vibrational bands. The experimental results, combined with classical molecular dynamics simulations with a force field effectively accounting for electronic polarization via charge scaling and ab initio simulations, indicate that the measured binding constants pertain to direct (as opposed to water separated) ion pairing. The
\end{abstract}


resulting binding constants do not scale with cation size, as the binding constant to $\mathrm{Zn}^{2+}$ is significantly larger than that to either $\mathrm{Mg}^{2+}$ or $\mathrm{Ca}^{2+}$, although $\mathrm{Zn}^{2+}$ and $\mathrm{Mg}^{2+}$ have similar radii that are about $25 \%$ smaller than $\mathrm{Ca}^{2+}$. Remaining uncertainties in the metal acetate binding free energies are linked to fundamental ambiguities associated with identifying the range of structures pertaining to non-covalently bound species. 


\section{Introduction}

Divalent non-transition metal cations, in particular calcium $\left(\mathrm{Ca}^{2+}\right)$, magnesium $\left(\mathrm{Mg}^{2+}\right)$, and zinc $\left(\mathrm{Zn}^{2+}\right)$, play important roles in many biological processes and diseases. ${ }^{1-10}$ Calcium is one of the key signaling species triggering changes in conformations of proteins involved, among others, in interactions between neurons, contraction of muscle cells, or exocytosis. ${ }^{1,2}$ Magnesium is vital, for example, for enzymatic catalysis including ATP synthesis and for stabilizing nucleic acids. ${ }^{3-5}$ Similarly, zinc is essential for proper function of many enzymes involved, e.g., in cell growth and reproduction, as well as for DNA stability and gene expression. ${ }^{6,7}$ The biological functions of these ions are realized by specialized ion-binding structures of the relevant biomolecules, such as the calcium-binding EF-hand motif ${ }^{8}$ and the zinc finger ${ }^{9}$ in proteins or phosphate-containing nucleic acid backbone motifs for binding of magnesium. ${ }^{10}$ Carboxylate of the side chain of glutamate or aspartate, or monovalent phosphate of the DNA/RNA backbone of phosphorylated biomolecules are examples of biologically relevant negatively charged groups that interact with divalent cations. Here, we focus on the former group with the aim to elucidate the structural and energetic details of the interaction between a carboxylate anion with $\mathrm{Ca}^{2+} / \mathrm{Mg}^{2+} / \mathrm{Zn}^{2+}$ in an aqueous environment.

Although one expects these cations to ion-pair with biochemically ubiquitous carboxylate groups, previous experimental studies have reached conflicting conclusions regarding the corresponding binding strengths to simple carboxylates such as acetate $\left(\mathrm{Ac}^{-}\right)$anions. In addition, molecular modeling of the interactions of dications with their biomolecular binding partners can only be as trustworthy as the underlying force field. It is, therefore, crucial to properly benchmark the interaction parameters against experimental data. ${ }^{11,12}$ Prior metal carboxylate binding constant compilations $^{13}$ and individual studies include experimental potentiometric, ${ }^{14-18}$ NMR, ${ }^{19}$ vibrational spectroscopic, ${ }^{20-22}$ and surface sum frequency ${ }^{23,24}$ measurements, as well as theoretical 
classical ${ }^{25}$ and quantum, ${ }^{26}$ calculations. The reported acetate binding constants for a given cation often vary by up to an order of magnitude, and are not always in agreement regarding the relative binding strengths of the above three cations (although studies that have included all three cations generally agree that $\mathrm{Zn}^{2+}$ binds more strongly to $\mathrm{Ac}^{-}$than either $\mathrm{Mg}^{2+}$ or $\left.\mathrm{Ca}^{2+}\right) \cdot{ }^{13,14}$

Most prior experimental studies of metal carboxylate binding constants have utilized potentiometric measurements. Although such measurements have provided a vast data base of binding constant results, ${ }^{94}$ they are inherently ambiguous with regard to the structures of the bounds species, and are susceptible to systematic errors associated with interfacial electrode effects. ${ }^{15}$ Vibrational spectroscopic methods offer an appealing alternative detection strategy, as the associated spectral changes contain molecularly detailed information pertaining to both the free and bounds species (and their hydration-shells). However, prior attempts to use vibrational infrared and Raman spectroscopy to determine metal acetate binding constants were restricted to the use of Gaussian-Lorentzian fits to the acetate CC stretch band (near $\left.940 \mathrm{~cm}^{-1}\right)^{21,22}$ and did not report consistent binding constant results for $\mathrm{Zn}^{2+}, \mathrm{Mg}^{2+}$ and $\mathrm{Ca}^{2+}$. These prior studies also concluded that the carboxylate $\mathrm{COO}^{-}$stretch band (near $1400 \mathrm{~cm}^{-1}$ ) was less quantitatively useful, presumably because of its overlap with to a $\mathrm{CH}_{3}$ deformation mode, ${ }^{27}$ although the apparent splitting between the symmetric and antisymmetric carboxylate stretch sub-bands has been interpreted as providing some information regarding the presence of unbound (free acetate), monodentate, bidentate, and bridging complexes..$^{20-22}$

In an effort to resolve these discrepancies we have used a new Raman multivariate curve resolution (Raman-MCR) measurement strategy to obtain self-consistent one-to-one $\mathrm{Ac}^{-} \cdots \mathrm{M}^{2+}$ binding free energies in aqueous $\mathrm{MAc}_{2}$ solutions (where $\mathrm{M}=\mathrm{Zn}, \mathrm{Mg}$, or $\mathrm{Ca}$ ). We demonstrate that a Raman-MCR based spectral decomposition strategy may be used to obtain robust metal acetate 
binding constants, without relying on any a priori assumptions regarding the shapes of the unbound and bound acetate spectra. Our results further demonstrate that self-consistent one-to-one binding constants may be obtained from aqueous $\mathrm{MAc}_{2}$ solutions with concentrations up to $0.5 \mathrm{M}$, using spectral decompositions of either the acetate $\mathrm{CC}$ or $\mathrm{COO}^{-}$bands (or both). These experimental conclusions are corroborated by our molecular dynamics (MD) simulations with a force field that effectively accounts for electronic polarization via charge scaling. Our MD results confirm that the binding affinities to the carboxylate group are not correlated with the cationic radii and also provide molecular insight into the ion pairing geometries. In particular, properly benchmarked simulations and quantum calculations allow us to address detailed structural and energetic issues concerning the modes of cation - carboxylate binding (i.e., contact vs. solventshared and, in the former case, monodentate vs. bidentate, see Figure 1), as deduced from spectroscopic measurements in this work as well as in previous studies. ${ }^{20,21,25,27-37}$

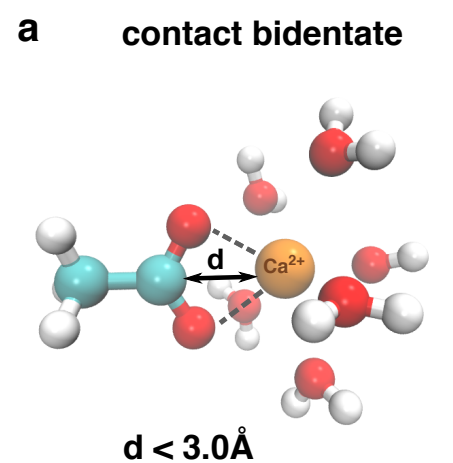

b monodentate

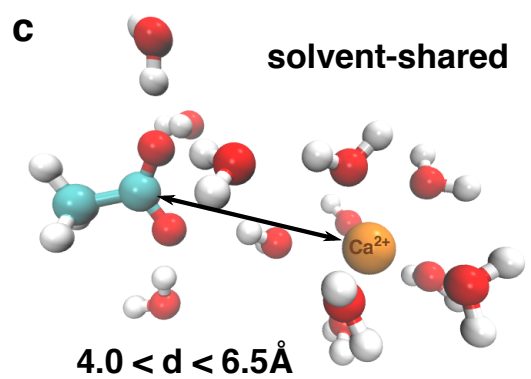

Figure 1. Sketch of the different cation-acetate ion pairing modes with the surrounding layer of water molecules, with typical associated $\mathrm{C}$-cation distances (for a $\mathrm{Ca}^{2+}$ cation). Snapshots were prepared using the VMD software. ${ }^{38}$ 


\section{Methods}

\subsection{Experimental details}

Sample preparation and Raman-MCR spectra. Sodium acetate (Mallinkrodt, 99+\%), magnesium acetate tetrahydrate (Sigma Aldrich, 99+\%), calcium acetate monohydrate (Sigma Aldrich, 99+\%), zinc(II) acetate dihydrate (Sigma Aldrich, 98+\%), and acetic acid (Sigma Aldrich, $99.7+\%)$ were used without further purification. Aqueous solutions of sodium acetate (NaAc), magnesium acetate $\left(\mathrm{MgAc}_{2}\right)$, calcium acetate $\left(\mathrm{CaAc}_{2}\right)$, and zinc(II) acetate $\left(\mathrm{ZnAc}_{2}\right)$ were prepared by adding an appropriate mass of each solid to a volumetric flask and completed with ultra-purified water (Milli-Q UF Plus, $18.2 \mathrm{M} \Omega \mathrm{cm}$, Millipore) to obtain stock solutions of $4 \mathrm{M}\left(\mathrm{Na}^{+}\right.$solutions), $2 \mathrm{M}\left(\mathrm{Mg}^{2+}\right.$ and $\mathrm{Ca}^{2+}$ solutions $)$, or $1.5 \mathrm{M}\left(\mathrm{Zn}^{2+}\right.$ solutions). Solutions were sonicated for $\sim 1 \mathrm{~min}$ to aid dissolution and were then diluted to obtain the desired concentrations.

Raman vibrational spectra were collected at $20^{\circ} \mathrm{C}$ using a home-built instrument with a $514.5 \mathrm{~nm}$ Argon ion laser and $20 \mathrm{~mW}$ of power at the sample. For each sample, two to four spectra were collected with an integration time of 5 min. Raman-MCR spectra were obtained using the self-modeling curve resolution (SMCR) algorithm. ${ }^{39-41}$ In this procedure, measured Raman spectra of pure water and solutions were decomposed to obtain minimum area non-negative solutecorrelated (SC) spectra, containing vibrational features arising from the solute itself and from the water molecules that are perturbed by the solute. Unless stated otherwise, all of the reported Raman-MCR solute-correlated (SC) spectra are normalized to the solute CC band area. This normalization produces spectra whose $\mathrm{CC}$ band area is concentration independent, which is also the case when normalizing the SC spectra to the corresponding solute concentration. The CC normalization procedure is more effective than concentration normalization in suppressing variations due to drifts in the excitation laser intensity or optical alignment. 
Ion-pairing binding constant determination. Raman-MCR SC spectra were used to determine the binding constants of acetate $\left(\mathrm{Ac}^{-}\right)$to metal dications. For a given aqueous $\mathrm{MAc}_{2}$ solution, the $\mathrm{SC}$ spectrum contains features arising from unbound $\mathrm{Ac}^{-}$, bound $\mathrm{Ac}^{-}$, and water molecules perturbed by these two acetate species as well as by the $\mathrm{M}^{2+}$ ions. Total least squares (TLS) analysis was applied to the SC spectra to estimate the concentrations of unbound $\mathrm{Ac}^{-}$and bound $\mathrm{Ac}^{-}$. This TLS procedure required obtaining the pure component spectra for the unbound and bound acetate species. The unbound component was chosen as the SC spectrum obtained from NaAc solutions (because the corresponding SC spectra are dominated by $\mathrm{Ac}^{-}$, as $\mathrm{Na}^{+}$has a negligible influence on water structure, ${ }^{42}$ and because there is little evidence of ion-pairing between $\mathrm{Na}^{+}$and $\mathrm{Ac}^{-}$at concentrations up to $2 \mathrm{M}$, as further described in the Results section). The bound component, which is not readily evident in the measured SC spectra, was obtained using a second SMCR decomposition in which the contributions of unbound $\mathrm{Ac}^{-}$(aqueous $\mathrm{NaAc}$ ) were removed from the SC spectra pertaining to each $\mathrm{MAc}_{2}$ solution (as described in the Supplementary Information). The resulting second round SMCR SC spectrum obtained contains vibrational features pertaining to the bound acetate $\mathrm{MAc}^{+}$species itself (as further described in the Results section).

\subsection{Computational details}

Molecular dynamics simulations and $\Delta \mathbf{G}_{\text {bind }}$ calculations. We used molecular dynamics simulations with different force fields to compute the binding free energy $\Delta G_{\text {bind }}$ of various cations $\left(\mathrm{Ca}^{2+}, \mathrm{Zn}^{2+}, \mathrm{Mg}^{2+}\right)$ to an acetate ion. Since sodium acetate is used experimentally as a non-binding reference, we also performed simulations with $\mathrm{Na}^{+}$. The simulation box contained one acetate anion, one cation $\left(\mathrm{Ca}^{2+}, \mathrm{Zn}^{2+}, \mathrm{Mg}^{2+}\right.$ or $\left.\mathrm{Na}^{+}\right)$and 1723 water molecules. The simulations were performed with the Gromacs 5.1.1 software, ${ }^{43}$ in the constant temperature/constant pressure (NpT) ensemble, using the Parinello-Rahman barostat ${ }^{44}$ with a 5 ps coupling time and the velocity 
rescaling thermostat ${ }^{45}$ at $300 \mathrm{~K}$ with a 1 ps coupling time. Periodic boundary conditions were used employing a Particle Mesh Ewald ${ }^{46}$ treatment of long-range electrostatic interactions with a $12 \AA$ cutoff. Hydrogen-containing bonds were constrained using the LINCS algorithm, ${ }^{47}$ and the SETTLE algorithm for water molecules. ${ }^{48}$

Water molecules were described by the SPC/E force field, ${ }^{49}$ and different force fields were tested for the ions. For the cations, we first tried force fields commonly used in the literature, including the Joung-Cheatham parameters for $\mathrm{Na}^{+},{ }^{50}$ the $12-6$ Lennard-Jones parameters for divalent cations suggested by the Merz group, ${ }^{51}$ the $\mathrm{Ca}^{2+}$ force field developed by the Netz group, ${ }^{52}$ the $\mathrm{Mg}^{2+}$ force field suggested by $\mathrm{D}$. Tobias and coworkers, ${ }^{53}$ and the $\mathrm{Zn}^{2+}$ force field by Stote and Karplus. ${ }^{54}$ A previously derived Amber99-like force field was employed for acetate. ${ }^{11}$

Standard full charge force fields are known to overestimate ion pairing, especially with divalent cations because they miss part of the charge shielding provided by solvent polarization effects. Therefore, we also tested different "scaled charge" force fields, that follow the so-called Electronic Continuum Correction (ECC) theory, which effectively takes the electronic polarization effects into account, in a mean field approach. ${ }^{55-57}$ We tested previously developed ECC force fields for acetate ${ }^{11}$ and for $\mathrm{Na}^{+},{ }^{58} \mathrm{Ca}^{2+},{ }^{11,59} \mathrm{Mg}^{2+}$ and $\mathrm{Zn}^{2+12}$. We also used a "milder" scaling factor of 0.8 which assumes that part of the electronic polarization is effectively wrapped into the employed empirical water model, whose dielectric constant is larger than what would correspond solely to nuclear polarization. All the employed force field parameters are provided as GROMACS parameter files as Supplementary Data. ${ }^{60}$

Binding free energy calculations were performed within GROMACS following the wellestablished double-decoupling alchemical procedure.${ }^{61-64}$ In a first simulation (S1), the cation in pure water is progressively decoupled from its environment $\left(\Delta G_{1}\right)$; in a second simulation (S2), 
the cation in a specific ion pair geometry with acetate is progressively decoupled from its environment $\left(\Delta G_{2}\right)$. This was done first turning off the electrostatic interactions between the cation and its environment, before turning off the van der Waals interactions, using soft-core potentials to improve convergence, for a total of 24 windows. Starting from the coupled state, each window was equilibrated for $250 \mathrm{ps}$ before starting the next one, and another $10 \mathrm{~ns}$ sampling were then added in each window independently. A typical input file for one window is provided as Supplementary Data. ${ }^{60}$ The free energy difference associated with each alchemical transformation was reconstructed using the Bennett Acceptance Ratio ${ }^{65}$ (BAR) method as implemented in Gromacs. ${ }^{43}$ The error bar provided on $\Delta G_{\text {bind }}^{\text {o }}$ is the sum of the error on each free energy term $\Delta G_{1}$ and $\Delta G_{2}$, each being estimated from the average variance over 5 blocks as implemented by default in "gmx bar". As extensively discussed in the literature, several corrections need to be estimated

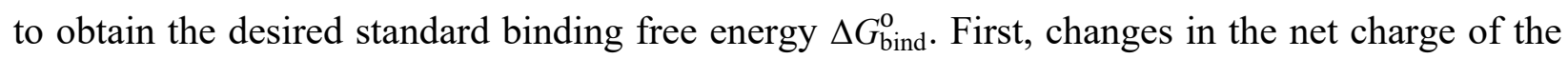
simulation box during ion decoupling induce a change in the neutralizing charge density, or "gellium", when using periodic boundary conditions and Particle Mesh Ewald summation. The interactions with the gellium contribute to the calculated decoupling free energies. ${ }^{66-68}$ Analytic estimates for the correction $\Delta G_{\mathrm{PBC}}$ have been suggested, ${ }^{68}$ and we use here the second order expansion (Eq. 17 from Ref ${ }^{68}$ ). Second, the binding geometry of the ion pair in the simulations S2 (contact monodentate, contact bidentate, solvent-shared) was enforced by a flat-bottomed harmonic restraint on the distance between the cation and the carbon of the carboxylate group. The details of the employed restraint for each cation are provided in the Supplementary Information (Table S2). The free-energy contribution of this restraint needs to be carefully accounted for both in the coupled and the uncoupled state. ${ }^{69-71}$ In the uncoupled state, the free energy of applying the restraint with respect to the standard state concentration can be expressed and numerically 
estimated as $\Delta G_{\text {restr }}=\int_{0}^{\infty} 4 \pi r^{2} \mathrm{e}^{-\beta U_{\text {restr }}(r)} \mathrm{dr} \approx \int_{0}^{L} 4 \pi r^{2} \mathrm{e}^{-\beta U_{\text {restr }}(r)} \mathrm{dr}$, where $L$ is the box size and $U_{\text {restr }}$ the flat-bottomed restraining potential. In practice the geometry and size of the box are irrelevant provided that $U_{\text {restr }} \gg k T$ for $r_{\max }<r<L$. In the coupled state, the free energy contribution due to the removal of the restraint can be expressed as $\Delta G_{\text {unrestr }}=-k T \ln \left\langle e^{+\beta U_{\text {restr }}}\right\rangle$, numerically estimated on the fully coupled simulation window. ${ }^{69}$ Finally, the standard binding free energy is obtained as $\Delta G_{\text {bind }}^{\mathrm{o}}=\Delta G_{1}-\Delta G_{2}+\Delta G_{\mathrm{PBC}}+\Delta G_{\mathrm{restr}}+\Delta G_{\mathrm{unrestr}}$. Note that the correction term $\left(\Delta G_{\mathrm{PBC}}+\Delta G_{\mathrm{restr}}+\Delta G_{\mathrm{unrestr}}\right)$ is here overall of the same magnitude as the raw free energy difference, and it is thus crucial to properly take it into account. The total binding free energy for contact pairs $\Delta G_{\text {bind,tot }}^{\mathrm{o}}$ is obtained by combining the binding free energy in each ion pair geometry, monodentate $\Delta G_{\text {bind,mono }}^{\mathrm{o}}$ and bidentate $\Delta G_{\text {bind,bi }}^{\mathrm{o}}$ as follows:

$$
\Delta G_{\text {bind,tot }}^{\mathrm{o}}=-R T \ln \left(e^{-\Delta G_{\text {bind,mono }}^{\mathrm{o}} / R T}+e^{-\Delta G_{\text {bind,bi }}^{\mathrm{o}} / R T}\right)
$$

A detailed example for one cation in one specific binding geometry is provided in the Supplementary Information.

When decoupling a cation from acetate in a monodentate binding mode, the configurational distribution of the bound state is strongly bimodal, and symmetric: it is composed of two equivalent binding modes involving each carboxylate oxygen atom. Each of these two modes is potentially metastable on the time scale of simulations, therefore the initial windows of the decoupling transformation are very likely to present incomplete sampling, unevenly distributed between the two modes. Our numerical tests indicate that this is the case with most of the full charge force fields and some scaled charge (esp. for $\mathrm{Mg}^{2+}$ and $\mathrm{Zn}^{2+}$ ), however, due to the symmetry of the energy distribution, this does not introduce a bias, or even significant added variance, in our free energy estimates. See Supplementary text and Figure S4 for details. 
Ab initio molecular dynamics simulations and free energy profiles. We computed the free energy profiles along the distance between the carboxylate carbon atom and the different cations using Born-Oppenheimer ab initio molecular dynamics (AIMD) simulations, performed with the Quickstep module of the CP2K 3.0 package $^{72}$ with the hybrid Gaussian and plane waves (GPWs) method. We limited our simulations to the range of distances corresponding to the contact bidentate-monodentate ion pair conformations, because the limited sampling allowed by AIMD simulations made it impossible to converge the free energy profiles at larger distances. The simulation box contained one cation $\left(\mathrm{Ca}^{2+}, \mathrm{Mg}^{2+}\right.$ or $\left.\mathrm{Zn}^{2+}\right)$ and one formate anion (smaller than acetate for these very expensive simulations) together with 106 water molecules, the box size being determined from the average box size in NpT simulations of the same system with a classical force field. The simulations were run in the NVT ensemble at $300 \mathrm{~K}$ using a velocity rescaling thermostat $^{45}$ (CSVR) with a 50 fs time constant. We used the BLYP ${ }^{73,74}$ density functional with the $\mathrm{D} 3 \mathrm{M}$ dispersion correction ${ }^{75,76}$ and a Becke-Johnson damping scheme, ${ }^{77} \mathrm{D} 3 \mathrm{M}(\mathrm{BJ})$. The same calculations were repeated with the older D2 dispersion correction ${ }^{78}$ to assess the robustness of the results with respect to the dispersion scheme. The electron density was described with the DZVPMOLOPT-SR-GTH double- $\zeta$ basis set, ${ }^{79}$ with GTH pseudopotentials ${ }^{80}$ for the core electrons and a 400 Ry cutoff for the auxiliary plane-wave basis. We used a total of 10-15 umbrella sampling windows (depending on the cation) with the distance of interest restrained around values varying from $2.3 \AA$ (for $\mathrm{Mg}^{2+}$ and $\mathrm{Zn}^{2+}$ ) or $2.7 \AA$ (for $\mathrm{Ca}^{2+}$ ) to $3.75 \AA$. Each window was started from an equilibrated configuration obtained from a classical simulation with the same restraining potential. In the barrier region between the two binding modes, we used several starting points with different starting geometries with typical hydration patterns. We accumulated 40 ps of trajectory in each window, after a 5 ps equilibration. Each free energy profile thus aggregates around 500 ps of 
AIMD simulations. Free energy profiles were obtained from simulation trajectories using WHAM, ${ }^{81}$ as implemented in ref ${ }^{82}$. Typical input files are provided as Supplementary Data. ${ }^{60}$

Raman spectra modeling. We took 200 snapshots from the MD simulations performed in each ion pair geometry for each cation, as described for the binding free energy calculations. For each snapshot, we extracted the acetate and the bound ion, together with the water molecules that have at least one atom within $1.2 \mathrm{~nm}$ from either cation or acetate. The geometry of each snapshot was then optimized before calculation of the Raman spectrum. All calculations were done using the Gaussian 16 package..$^{83}$ The acetate and the bound ion were described at the B3LYP/6-31++G** level of theory, while the first layer of water molecules (any water molecule with an atom closer than $0.3 \mathrm{~nm}$ from any atom of acetate/cation) was described at the B3LYP/6-31G* level of theory. The water molecules in the second layer (MM region, $0.3-0.6 \mathrm{~nm}$ cutoff) were described using the TIP3P force field ${ }^{84}$ and the coupling between the QM and MM regions was modeled using ONIOM method $^{85}$ with electrostatic embedding. The last layer of water molecules $(0.6-1.2 \mathrm{~nm})$ was described only as partial atomic charges. Each snapshot then underwent a 10-step optimization prior spectra calculation, where the acetate, the cation, and QM layer of water molecules were allowed to move, while the second layer of water molecules and point charges remained frozen. After this optimization procedure, the vibrational frequencies and Raman intensities (SCP modulation scheme with backscattering detection $\left(180^{\circ}\right)$ ) were calculated using the harmonic approximation, together with far from resonance CPKS theory ${ }^{86}$ at $532 \mathrm{~nm}$. Then, the signal originating from water molecules was deleted using an in house developed script and the resulting intensities were broadened using a Lorentzian function with a full width at half maximum of $7.5 \mathrm{~cm}^{-1}$ and corrected for the temperature factor at $300 \mathrm{~K} .{ }^{87}$ The small difference between the simulation (i.e., $532 \mathrm{~nm}$ excitation frequency and $300 \mathrm{~K}$ ) and experimental conditions are expected 
to have a negligible effect on the spectra, so that both results sets can be directly compared. Finally, the spectra of all individual snapshots were averaged to yield the final averaged Raman spectra.

\section{Results}

Raman spectroscopy. Figure 2A shows Raman spectra of aqueous acetic acid and sodium acetate at similar concentrations. The major peaks in these spectra arise from the solute intra-molecular $\mathrm{CC}$ (near $\left.900 \mathrm{~cm}^{-1}\right), \mathrm{COO}^{-}\left(\right.$near $\left.1500 \mathrm{~cm}^{-1}\right)$, and $\mathrm{CH}\left(\right.$ near $2900 \mathrm{~cm}^{-1}$ ) vibrational modes, as well as an $\mathrm{OH}$ band (near $3400 \mathrm{~cm}^{-1}$ ) resulting primarily from water molecules. Comparison of the acetic acid and sodium acetate SC spectra reveals that deprotonation of carboxylic acid ${ }^{88,89}$ induces a significant redshift in the $\mathrm{CO}$ stretch band (from $\sim 1700 \mathrm{~cm}^{-1}$ for $-\mathrm{COOH}$ to $\sim 1400 \mathrm{~cm}^{-1}$ for $-\mathrm{COO}^{-}$), as well as smaller shifts in the solute $\mathrm{CC}$ and $\mathrm{CH}$ bands. The observed redshift of the acetate $\mathrm{COO}^{-}$band (relative to the acetic acid $\mathrm{COOH}$ band) is consistent with the fact that the extra electron is added to an antibonding orbital of the anion. ${ }^{89,90}$ Additionally, the larger intensity of the acetate $\mathrm{COO}^{-}$band implies that the extra electron increases the corresponding polarizability derivative. Note that the acetic acid and acetate stretch $\mathrm{CO}$ bands overlap with smaller bands (shoulders) near $1350 \mathrm{~cm}^{-1}$ assigned to a $\mathrm{CH}_{3}$ deformation mode. ${ }^{33}$ 

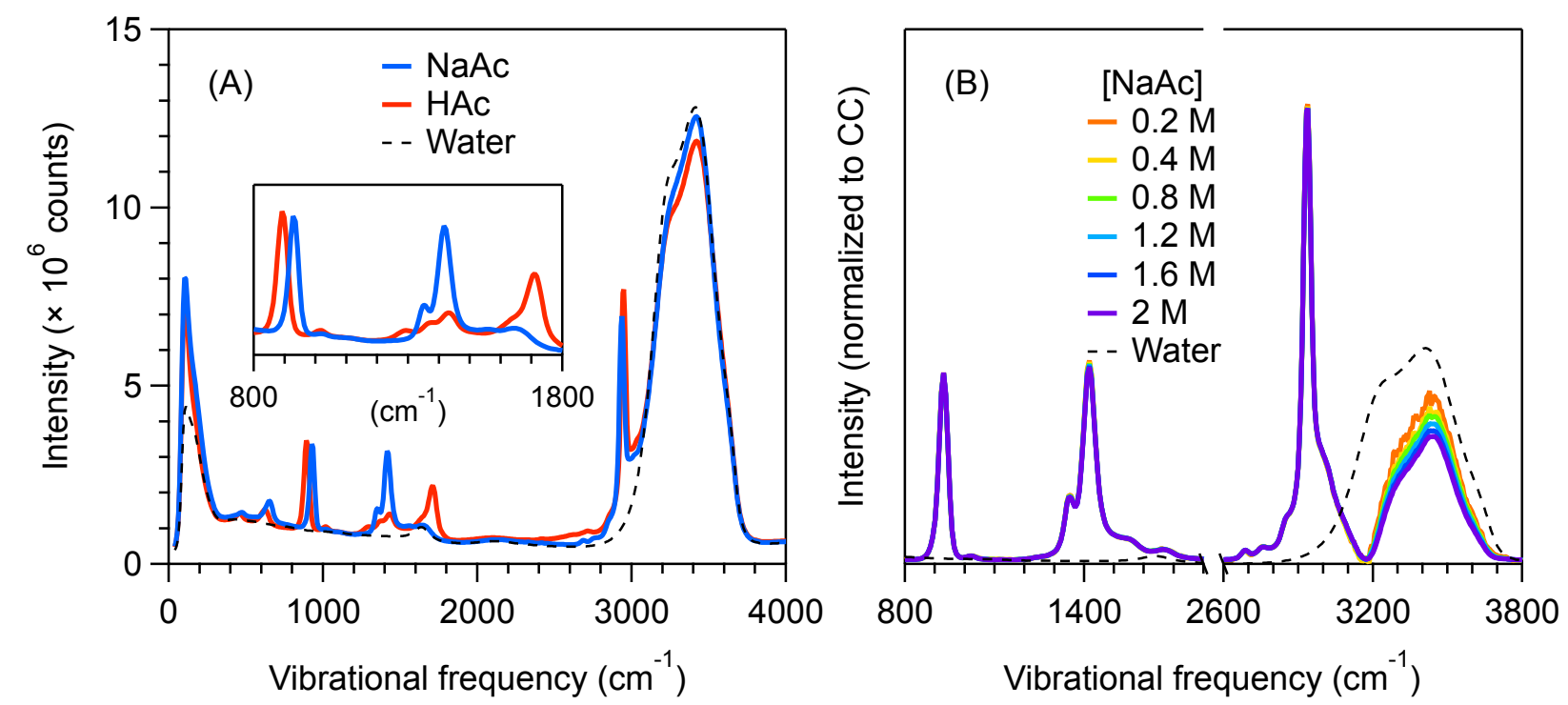

Figure 2. (A) Raman spectra of acetic acid and sodium acetate at approximately equal concentrations. (B) Raman-MCR SC spectra of sodium acetate solutions as a function of concentration. The water spectrum (dashed black) is for reference.

Figure 2B shows how the Raman-MCR SC spectra of sodium acetate vary with concentration (after normalization to the $\mathrm{CC}$ band area). These results indicate that there is essentially no concentration dependent shift or shape changes in the $\mathrm{CC}, \mathrm{COO}^{-}$, or $\mathrm{CH}$ bands in aqueous NaAc solutions, thus suggesting that there are no direct contacts between $\mathrm{Na}^{+}$and $\mathrm{COO}^{-}$ up to concentrations of $\sim 2 \mathrm{M}$ or that any direct contacts that are present do not significantly influence the acetate intramolecular vibrational modes. The only readily detectable concentrationdependent change in the aqueous NaAc Raman-MCR spectra is the slight decrease in the intensity of the NaAc Raman-MCR SC OH stretch band with increasing NaAc concentration, shown in Figure 2B. This decrease in the $\mathrm{OH}$ band area suggests the presence of some water-separated contacts between $\mathrm{Na}^{+}$and $\mathrm{Ac}^{-}$, as would be expected to occur even in a nominally non-ion-pairing mixture of $\mathrm{Na}^{+}$and $\mathrm{Ac}^{-}$at concentrations above $\sim 1 \mathrm{M}$. 
Given the negligible concentration dependence of the acetate bands in aqueous NaAc Raman spectra, the SC spectrum of NaAc may be used as a free acetate reference to uncover evidence of acetate-dication binding in aqueous of $\mathrm{MAc}_{2}$ solutions. Specifically, Figure 3 shows that the Raman-MCR SC spectra of $\mathrm{MgAc}_{2}, \mathrm{CaAc}_{2}$, and $\mathrm{ZnAc}_{2}$ solutions are shifted with respect to that of aqueous NaAc, thus implying the presence of significant ion-pairing of acetate to $\mathrm{Mg}^{2+}$, $\mathrm{Ca}^{2+}$, and $\mathrm{Zn}^{2+}$ over this concentration range. Our assignment of the shift primarily to direct (as opposed to water separated) ion paring is supported by the fact that the magnitude of the observed blue-shift of the $\mathrm{CC}$ stretch band is consistent with that which is predicted by quantum calculations to occur upon direct contact-ion pair formation, as further described in the Spectra Modeling section.

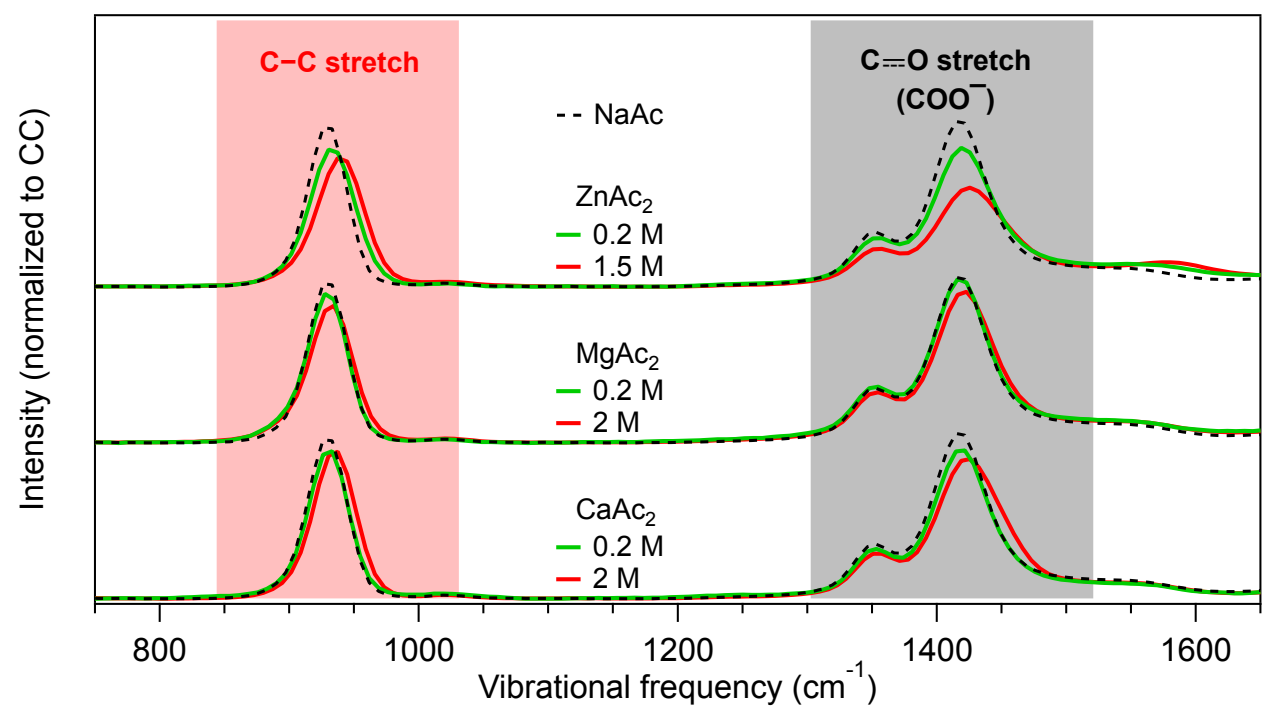

Figure 3. Concentration-dependent SC spectra of zinc(II) acetate (top), magnesium acetate (middle), and calcium acetate (bottom). The larger concentration dependent changes in the $\mathrm{CC}$ and $\mathrm{COO}^{-}$stretching band

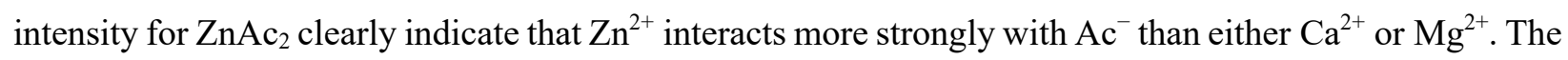
dashed black spectrum corresponds to aqueous are included as the unbound acetate reference spectrum. The spectra are vertically offset for clarity. 
The results in Figure 3 further suggest that direct-contact ion-pairing of acetate to $\mathrm{Zn}^{2+}$ produces a larger spectral change than binding to either $\mathrm{Ca}^{2+}$ or $\mathrm{Mg}^{2+}$, as evidenced by the larger concentration-dependent changes in the SC spectra of $\mathrm{ZnAc}_{2}$ than either $\mathrm{MgAc}_{2}$ and $\mathrm{CaAc}_{2}$ at the same concentration. A quantitative experimental estimate of the corresponding binding constants can, in principle, be obtained by decomposing the measured spectra into a linear combination of the unbound and bound acetate spectra. This procedure requires knowing a priori the SC spectra of these two pure component acetate species. The spectrum of aqueous NaAc yields the unbound $\mathrm{COO}^{-} \mathrm{SC}$ spectrum (as previously discussed). The spectrum of the bound acetate species is more difficult to unambiguously determine. However, we have found that a self-consistent estimate of this spectrum may be obtained using a second round SMCR procedure in which the contribution of unbound acetate (obtained from the aqueous $\mathrm{SC}$ spectrum of $\mathrm{NaAc}$ ) is removed from the $\mathrm{SC}$ spectra of aqueous $\mathrm{MAc}_{2}$ solutions (as further described in the Supplementary Information). Briefly, the second round SMCR analysis produces a family of possible bound spectra, but only one member of this family is found to produce a self-consistent (concentration-independent) oneto-one binding constant over the experimental concentration range of $0.1 \mathrm{M}$ to $0.5 \mathrm{M} \mathrm{MAc}_{2}$. The remaining members of the family of possible bound spectra include a mathematical lower bound minimum area spectrum (pertaining to the most tightly bound ion-pairs with the smallest possible binding constant), as well as a distribution of other possible bound spectra whose shape approaches that of the free acetate (pertaining to the most loosely bound ion-pairs with the largest binding constants). Although all of these bound spectra are mathematically possible, only one yields the self-consistent one-to-one binding constants between acetate and each of the three cations. The robustness of the resulting binding constant is confirmed by very similar results obtained when performing the TLS spectral decomposition using only the $\mathrm{CC}$ or $\mathrm{COO}^{-}$bands or by 
simultaneously fitting both bands (as further discussed below and in the Supplementary Information).

Figure 4A-C shows the free (dashed black) and bound (dashed red) acetate spectra, obtained as described above (and in the Supplementary Information), and the corresponding quantitative decomposition of the aqueous $\mathrm{MAc}_{2}$ solution spectra, along with the measured SC spectrum for an aqueous $0.5 \mathrm{M} \mathrm{MAc}_{2}$ solution (green curve). The inset spectra on the right-hand panels show the essentially perfect agreement between the TLS reconstructions (dotted black curves) and the measured $0.5 \mathrm{M} \mathrm{SC}$ spectra (solid green curves), where the TLS spectra represent a best-fit of the measured spectrum to a linear combination of the unbound and bound spectra. The yellow points in the right-hand panels in Figure 4 show the resulting bound acetate fraction obtained for solutions with $\mathrm{MAc}_{2}$ concentrations up to $0.5 \mathrm{M}$, and thus total acetate concentrations up to $1 \mathrm{M}$. Note that these binding constants imply that $\mathrm{Zn}^{2+}$ has a binding constant to acetate $(K=4.5 \pm 1.3)$ that is about four times larger than $\operatorname{Mg}^{2+}(K=1.1 \pm 0.4)$ and $\mathrm{Ca}^{2+}(K=1.1 \pm 0.4)$. 

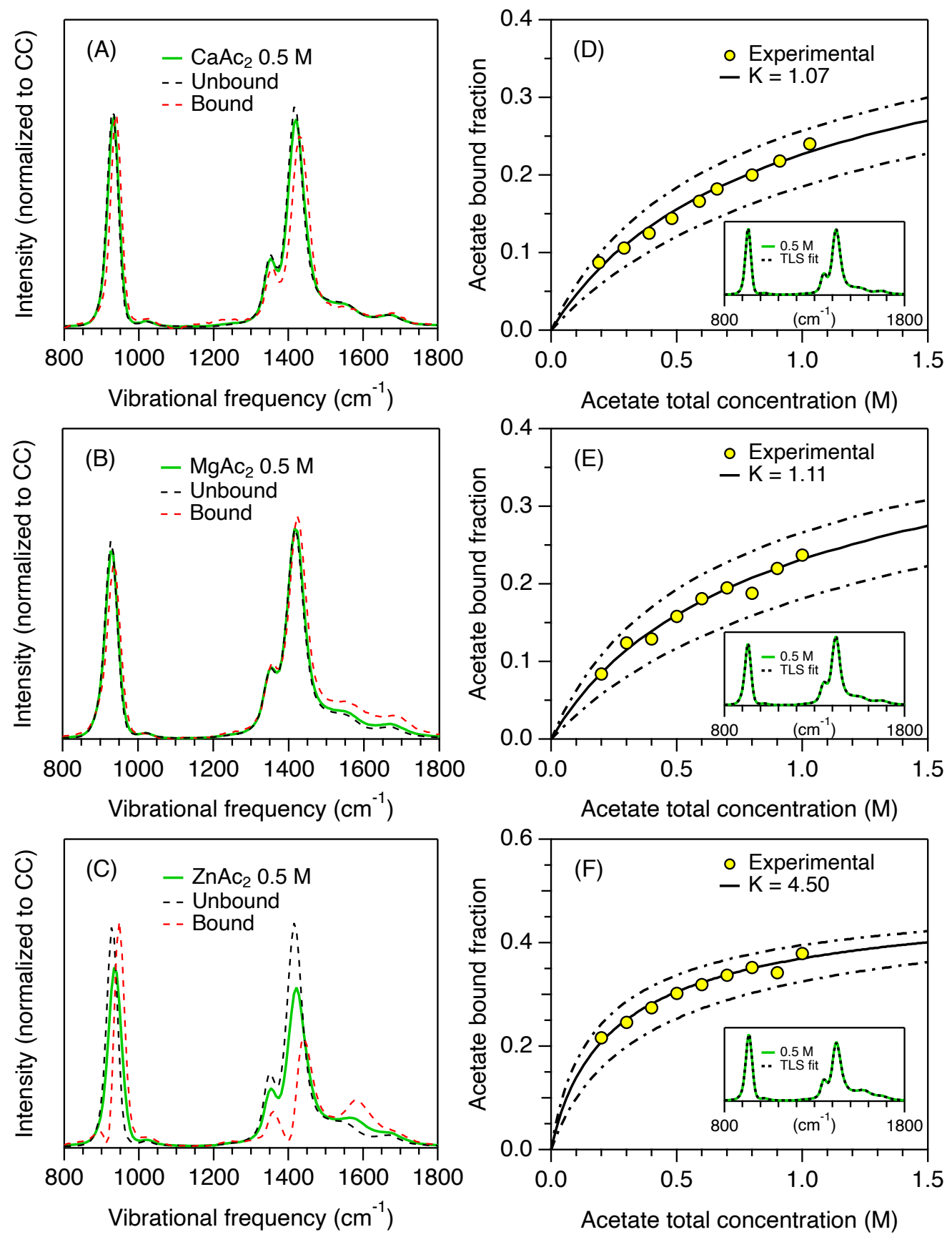

Figure 4. Free and bound component spectra (left-hand panels A-C) and binding curves (right-hand panels D-F) for magnesium (A and D), calcium (B and E) and zinc (C and F). The inset spectra in panels D-F compare the TLS reconstruction (dotted black curves) with the experimental SC spectrum (solid green curves) obtained for a $0.5 \mathrm{M}$ solution of $\mathrm{MAc}_{2}$. The dot-dashed curves represent a $99 \%$ confidence interval for the binding constants. 
Spectra modeling. While the previously described Raman experiments provide an experimental measure of the binding constant between divalent cations and acetate, they cannot determine the geometry of the observed ion pairs and have a quite limited ability to distinguish different types of ion pairs: contact monodentate, contact bidentate or solvent shared ion pairs. In order to determine which type of ion pairs can lead to a shift in the measured Raman spectra, we computed the spectra (Figure 5) of acetate involved in different ion pair configurations with $\mathrm{Ca}^{2+}, \mathrm{Mg}^{2+}, \mathrm{Zn}^{2+}$, and $\mathrm{Na}^{+}$using quantum chemical (DFT-based) calculations on a large number of snapshots extracted from molecular dynamics simulations (see Methods). Although the shape of the band near $\sim 1450 \mathrm{~cm}^{-1}$ is not perfectly reproduced, both peaks $\left(\sim 950 \mathrm{~cm}^{-1}\right.$ and $\left.\sim 1450 \mathrm{~cm}^{-1}\right)$ exhibit a blue shift when in contact ion pair with $\mathrm{Ca}^{2+}, \mathrm{Mg}^{2+}$, and $\mathrm{Zn}^{2+}$. This is more pronounced for the bidentate ion pair and for $\mathrm{Zn}^{2+}$ and $\mathrm{Mg}^{2+}$ than $\mathrm{Ca}^{2+}$, which is in very good agreement with the experiment, and thus validates the procedure used to extract the signal of the bound acetate from the raw spectra.

The first important finding is that the simulated spectra show that no significant shift is observed between the reference spectrum of acetate in pure water, and that of acetate ion paired with $\mathrm{Na}^{+}$(see Supplementary Information). This validates the use of the sodium acetate solution as the "unbound" reference in the experiment. It also shows that even if there was significant pairing between acetate and sodium cations, the Raman experiment would not be able to capture it. The computed spectra for the contact ion pairs with $\mathrm{Ca}^{2+}, \mathrm{Mg}^{2+}$, and $\mathrm{Zn}^{2+}$ exhibit a blue-shift in the CC stretch band (near $940 \mathrm{~cm}^{-1}$ ) that is more pronounced for $\mathrm{Zn}^{2+}$ and $\mathrm{Mg}^{2+}$ than $\mathrm{Ca}^{2+}$ and are larger for the bidentate than the monodentate ion pairs. It is also clear that the formation of solventshared ion pairs (for all cations) does not significantly affect the peak positions. Hence, the experimental binding-induced spectral changes are evidently due primarily to the formation of 
direct contact ion pairs. The fact that the predicted $\mathrm{Zn}^{2+} \ldots \mathrm{Ac}^{-}$bidentate shift of $\sim 30 \mathrm{~cm}^{-1}$ is comparable to the experimentally observed shift of $\sim 20 \mathrm{~cm}^{-1}$ suggests that the experimental $\mathrm{ZnAc}_{2}$ solutions may include bidentate bound species (over the experimental concentration range up to $0.5 \mathrm{M})$, probably in addition to monodentate ones. In contrast, the smaller experimental bindinginduced $\mathrm{CC}$ band shifts of $\sim 10 \mathrm{~cm}^{-1}$ for $\mathrm{Mg}^{2+}$ may imply that the binding is primarily monodentate over the experimental concentration range. $\mathrm{For} \mathrm{Ca}^{2+}$, the calculated shifts for bidentate and monodentate ion pairs are much more similar, so that no clear conclusion regarding the contact ion pair structure can be drawn.
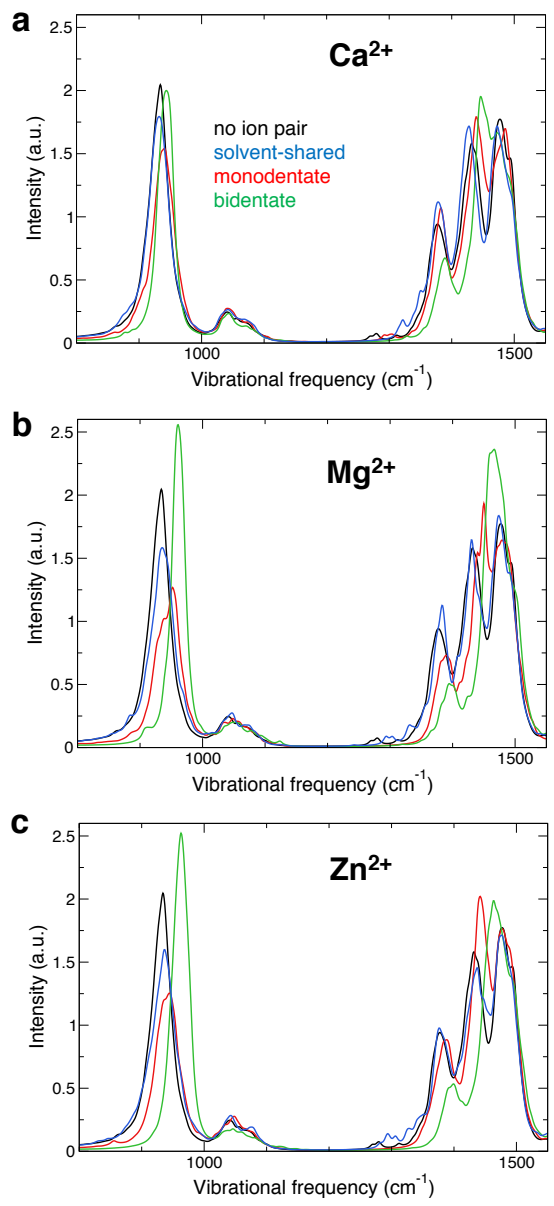

Figure 5. Simulated Raman spectra for acetate in pure water (black) or involved in a contact monodentate (red), contact bidentate (green) or solvent-shared (blue) ion pair with a a) calcium, b) magnesium and c) zinc cation. 
Binding constants and ion pair geometries. Since Raman measurements are primarily sensitive to direct contact ion pairs, they cannot assess the relative stability of contact vs solvent shared ion pairs or provide detailed information about the preferential mode of binding (monodentate or bidentate). Hence, molecular dynamics simulations can help complement the picture by providing access to the molecular details of the interaction and assess the relative stability of different ion pair structures.

However, standard nonpolarizable force fields are known to suffer from severe overbinding artefacts to describe the interaction between divalent cations and negatively charged moieties. ${ }^{11,91}$ These artefacts essentially originate from the lack of electronic polarization in such force fields. Several schemes have been suggested to correct this limitation, either through explicit inclusion of electronic polarization with the development of fully polarizable force fields (e.g. ${ }^{69,92-}$ ${ }^{95}$ ), or through a mean-field approach, the Electronic Continuum Correction, ${ }^{55-57,59}$ that suggests to implicitly account for electronic polarization by scaling the charge of charged moieties by a factor of $1 / \sqrt{\varepsilon_{\mathrm{el}}}=0.75$, where $\varepsilon_{\mathrm{el}}=1.78$ is the electronic part of the water dielectric constant. A milder factor 0.8 has also been tested as an attempt to compensate the fact water models have a dielectric constant that already contains more than pure nuclear polarization (see Methods). In a first step, we thus assessed the quality of different force fields-nonpolarizable with either full or scaled charges, and polarizable, with the AMOEBA force field ${ }^{92}$ - against the experimentally determined binding constants. Table 1 summarizes the obtained results, showing the range of binding free energy values in the cases where several force fields were tested. The uncertainty on 
each value is on the order of $0.5 \mathrm{~kJ} \mathrm{~mol}^{-1}$, and the details of the tested force fields and associated binding free energies are provided in the Supplementary Information (Table S4-S7).

It is important to notice that the Raman-derived binding constants for the contact ion pairs (or equivalently binding free energies) are obtained at finite ionic strength (between $\sim 0.3$ and $\sim 1.5 \mathrm{M}$ ), while calculated binding free energies correspond to a standard binding free energy in the infinite dilution limit. Hence, to compare measured and calculated binding free energies, we first extrapolated the experimentally-derived binding free energies to zero ionic strength, using a refined Davies ${ }^{96}$-like equation fitted on a large set of ion-ion binding constants measured at different ionic strengths. ${ }^{97} \mathrm{We}$ thus report in Table 1 the extrapolated experimental binding free energy, which can be directly compared with the computed contact binding free energy, that encompasses both monodentate and bidentate binding geometries. Note that these extrapolated binding free energies are still quite small, around $-3.2 \mathrm{~kJ} \mathrm{~mol}^{-1}$ for $\mathrm{Ca}^{2+},-3.4 \mathrm{~kJ} \mathrm{~mol}^{-1}$ for $_{\mathrm{Mg}^{2+}}$, and only $-6.9 \mathrm{~kJ} \mathrm{~mol}^{-1}$ for $\mathrm{Zn}^{2+}$.

Table 1. Standard binding free energy of different divalent cations with acetate at zero ionic strength as extrapolated from the Raman experiments, and calculated for different ion pair geometries using either a full charge force field, an ECC force field with the usual 0.75 scaling factor or with a 0.8 scaling factor, and with the fully polarizable AMOEBA force field. The details of the employed force fields and corresponding binding free energies are provided in the Methods section and Supplementary Information (Sections 2 and 3, Tables S4-S7). When different force fields were tested, the two values are separated with /. The error bar on all calculated values is about $0.5 \mathrm{~kJ} \mathrm{~mol}^{-1}$.

\begin{tabular}{|c|c|c|c|c|c|}
\hline $\begin{array}{c}\Delta \mathrm{G}_{\text {bind }}{ }^{\circ} \\
\left(\mathrm{kJ} \mathrm{mol}^{-1}\right)\end{array}$ & $\begin{array}{c}\text { Experimental } \\
\quad(I=0)\end{array}$ & $\begin{array}{c}\text { Full charge } \\
\text { FF }\end{array}$ & $\begin{array}{c}\text { ECC FF } \\
\text { (scaling 0.75) }\end{array}$ & $\begin{array}{c}\text { ECC FF } \\
\text { (scaling 0.8) }\end{array}$ & AMOEBA \\
\hline \multicolumn{6}{|l|}{$\mathrm{Ca}^{2+}$} \\
\hline $\begin{array}{c}\text { Contact } \\
\text { bidentate }\end{array}$ & n.a. & $-12.3 /-21.7$ & $+5.4 /+6.3$ & +2.5 & -2.0 \\
\hline $\begin{array}{c}\text { Contact } \\
\text { monodentate }\end{array}$ & n.a. & $-10.1 /-18.4$ & $+2.0 /-0.6$ & 0.0 & -11.3 \\
\hline Total contact & $-3.2 \pm 0.2$ & $-13.2 /-22.3$ & $+1.5 /-0.7$ & -0.8 & -11.4 \\
\hline
\end{tabular}




\begin{tabular}{|c|c|c|c|c|c|}
\hline $\begin{array}{c}\text { Solvent- } \\
\text { shared }\end{array}$ & n.a. & -6.7 & $-2.5 /-3.1$ & -2.7 & -1.7 \\
\hline \multicolumn{6}{|l|}{$\mathrm{Mg}^{2+}$} \\
\hline $\begin{array}{l}\text { Contact } \\
\text { bidentate }\end{array}$ & n.a. & $-29.9 /-13.0$ & $+18.1 /+19.7$ & +15.6 & n.a. \\
\hline $\begin{array}{c}\text { Contact } \\
\text { monodentate }\end{array}$ & n.a. & $-34.1 /-37.4$ & $-0.6 /-0.1$ & -5.3 & -11.9 \\
\hline Total contact & $-3.4 \pm 0.4$ & $-34.5 /-37.4$ & $-0.6 /-0.1$ & -5.3 & -11.9 \\
\hline $\begin{array}{l}\text { Solvent- } \\
\text { shared }\end{array}$ & n.a. & $-6.6 /-6.8$ & $-3.4 /-1.1$ & -1.3 & -1.8 \\
\hline \multicolumn{6}{|l|}{$\mathrm{Zn}^{2+}$} \\
\hline $\begin{array}{l}\text { Contact } \\
\text { bidentate }\end{array}$ & n.a. & -14.9 & +21.4 & +19.4 & n.a. \\
\hline $\begin{array}{c}\text { Contact } \\
\text { monodentate }\end{array}$ & n.a. & -36.8 & +0.6 & -3.7 & n.a. \\
\hline Total contact & $-6.9 \pm 0.4$ & -36.8 & +0.6 & -3.7 & n.a. \\
\hline $\begin{array}{c}\text { Solvent- } \\
\text { shared }\end{array}$ & n.a. & -6.9 & -2.0 & -2.4 & n.a. \\
\hline
\end{tabular}

The binding free energy calculations show that nonpolarizable full charge force fields strongly overestimate the strength of the cation-acetate interaction, by at least $10 \mathrm{~kJ} \mathrm{~mol}^{-1}$ for $\mathrm{Ca}^{2+}$, and by about $30 \mathrm{~kJ} \mathrm{~mol}^{-1}$ for $\mathrm{Mg}^{2+}$ and $\mathrm{Zn}^{2+}$, and thus cannot be trusted to give a faithful representation of these binding processes. In contrast, ECC force fields with the standard 0.75 scaling factor predict much weaker binding constants, qualitatively in better agreement with the experiment, notably for $\mathrm{Mg}^{2+}$ and $\mathrm{Zn}^{2+}$, but quantitatively too weak by about $3-4 \mathrm{~kJ} \mathrm{~mol}^{-1}$. Since several works ${ }^{98,99}$ suggested that the use of a water force field that does not have the expected dielectric constant would justify using a scaling factor larger than 0.75 (but always smaller than 1), we performed simulations with test versions of the acetate and cation force fields using a scaling factor of 0.8 . This indeed in all cases improved the predicted binding free energy. However, the difference between the binding free energies for $\mathrm{Ca}^{2+}$ and $\mathrm{Mg}^{2+}$ is too large in the simulations (they are identical within error bars in the experiment), and none of the force fields describes the significantly stronger (by $3.5 \mathrm{~kJ} \mathrm{~mol}^{-1}$ ) binding with $\mathrm{Zn}^{2+}$ compared to the two other cations. For 
comparison, we also computed for $\mathrm{Ca}^{2+}$ and $\mathrm{Mg}^{2+}$ the binding free energy to acetate using the AMOEBA fully polarizable force field, which seems to overestimate the binding strength by about $8 \mathrm{~kJ} \mathrm{~mol}^{-1}$ for both cations. However, it does capture the almost identical binding constants of the two cations with acetate, which nonpolarizable force fields do not properly reproduce. Hence, even if the polarizable description still constitutes for $\mathrm{Mg}^{2+}$ a big improvement over full charge nonpolarizable force fields, the results are not in quantitative agreement with experimental data.

\section{Discussion}

Comparison with earlier experimental estimates of $\mathbf{K}$. Several estimates of the cation-acetate binding constants are available in the literature and can be compared to the presently obtained results (Table 2). Since the simulations effectively pertain to the zero ionic strength limit, we extrapolated all the values to zero ionic strength using a refined Davies-like equation ${ }^{97}$ (if the extrapolation was not already performed in the original work). Note that previous data have been obtained with potentiometric measurements, the reliability of which has been recently questioned. ${ }^{15}$ In addition, while we have shown that the present Raman-derived binding constants mainly report on the formation of contact ion pair, it is not clear how sensitive the potentiometric measurements are with respect to different ion pair geometries, even if one would expect it to be also mostly, but probably not exclusively, sensitive to contact ion pairs. Keeping these remarks in mind, our results show that $\mathrm{Zn}^{2+}$ binds about four times more strongly to acetate than either $\mathrm{Ca}^{2+}$ or $\mathrm{Mg}^{2+}$, in qualitative agreement with previous potentiometric measurements ${ }^{13,14}$ (and better quantitative agreement with more recently corrected potentiometric results ${ }^{15}$ for acetate binding to $\mathrm{Ca}^{2+}$ ). The difference between the binding constants of $\mathrm{Ca}^{2+}$ and $\mathrm{Mg}^{2+}$ is small and lies within the experimental error bars. This is in line with all previous studies that reported very similar binding 
constants for the two cations, either slightly in favor of one or the other, the difference probably being in each case within experimental error.

Table 2. Comparison of the standard binding constant $K^{\circ}$ at zero ionic strength for the formation of the 1:1 cation:acetate complex for different divalent cations, as obtained from present Raman experiments and from previous studies, all of which being extrapolated to zero ionic strength as described in ref ${ }^{97}$.

\begin{tabular}{|c|c|c|c|c|c|}
\hline & Present work & Ref ${ }^{14}$ & Ref ${ }^{13}$ & Ref $^{18}$ & Ref $^{15}$ \\
\hline $\mathrm{Ca}^{2+}$ & $3.6+/-0.4$ & 11.0 & $15.4+/-1.1$ & 10.4 & $2.7+/-0.3$ \\
\hline $\mathrm{Mg}^{2+}$ & $3.9+/-0.6$ & 10.3 & $18.6+/-1.0$ & 9.5 & n.a. \\
\hline $\mathrm{Zn}^{2+}$ & $16.0+/-2.8$ & 28.3 & 37.2 & n.a. & n.a. \\
\hline
\end{tabular}

Lessons learnt for ion pairing simulations. Comparing experimental binding estimates for the formation of ion pairs and values obtained with different force fields clearly highlighted the need to include electronic polarization to obtain a qualitatively correct picture, confirming previous studies. ${ }^{11,91}$ Standard nonpolarizable force fields systematically strongly overestimate the binding strength, by sometimes as much as $30 \mathrm{~kJ} \mathrm{~mol}^{-1}$, the error being more dramatic with small divalent ions such as magnesium and zinc. While explicit treatments of electronic polarization in force fields should be in principle better than the implicit mean-field charge scaling treatment used in the ECC approach, our results show that the former methods, at least in their current versions, do not always lead to better predictions than the ECC treatment. In some cases, such as the $\mathrm{Mg}^{2+}-$ acetate binding, the tested explicitly polarizable force field even performs significantly worse, even if it accurately predicts the same binding strength for calcium and magnesium. Hence, each force field should be thoroughly tested for the system and quantity of interest, with no guarantee that more elaborate force fields, in their current development state, provide more accurate results. Interestingly, for the ECC force field, in all the cases reported here, a 0.8 scaling performs 
significantly better than the standard "physically-sound" 0.75 scaling prescribed by the ECC theory. While this had been suggested before ${ }^{98}$ and can be rationalized in terms of limits of the available water models, this issue will be further investigated in future works.

Insights into binding patterns. Since our simulated spectra showed that the Raman experiments report mostly the formation of contact ion pairs, the present experiments demonstrate the existence of contact ion pairs between acetate and all the studied divalent cations. Even if the ratio between contact and solvent-shared ion pairs remains out of reach experimentally, our experimental and theoretical results imply that there is significant direct contact ion pair formation between acetate and the three dications $\left(\mathrm{Mg}^{2+}, \mathrm{Ca}^{2+}\right.$, and $\left.\mathrm{Zn}^{2+}\right)$ in aqueous $\mathrm{MAc}_{2}$ solution with concentrations down to $0.1 \mathrm{M}$. This conclusion is inconsistent with the assumption that some of these dications, such as $\mathrm{Mg}^{2+}$, always retains its hydration shell when ion-pairing with $\mathrm{COO}^{-}$.

In addition, our free energy calculations allow us to estimate the relative contact/solventshared binding, but the picture strongly depends on the details of the employed force field. For instance, while the AMOEBA polarizable force field predicts a very strong preference for the contact ion pairs with calcium, both ECC force fields predict the solvent-shared ion paired to be slightly more favorable, none of them quantitatively capturing the binding free energy. With the magnesium cation, both the 0.8-scaling ECC (in quantitative agreement with the experiment for the binding free energy) and the AMOEBA force fields exhibit a preference for the contact binding, even if the ratio (or equivalently, the free energy difference) with the solvent-shared geometry is quite different (larger with AMOEBA). Since none of the force fields captures the stronger binding with $\mathrm{Zn}^{2+}$ compared to the other cations, probably due to a missing partly covalent contribution, the details of its binding behavior can probably not be trusted in any classical simulation. 
Another controversial point in the literature is the nature of the favored contact binding mode for the different cations: monodentate (cation in direct contact with only one oxygen of the carboxylate group) or bidentate (cation in contact with both oxygen atoms). ${ }^{25,28,32,34,100,101}$ For $\mathrm{Mg}^{2+}$, all tested force fields, including the polarizable AMOEBA force field, give a consistent picture of the monodentate geometry being strongly favored. This is in agreement with the insights obtained from the comparison between measured and modeled spectral shifts (see Results section) and with several earlier reports, ${ }^{28,32,100,101}$ but conflicting with a previous study by some of us that suggested monodentate and bidentate modes to be both existing in solution. ${ }^{25}$ All force fields provide the same picture for $\mathrm{Zn}^{2+}$ as for $\mathrm{Mg}^{2+}$, with exclusive monodentate binding, but since they do not capture the specificity of zinc binding, they cannot be trusted to correctly describe binding modes. For $\mathrm{Ca}^{2+}$, the picture provided by the simulations is much less clear. While some force fields (incl. AMOEBA) predict the almost exclusive existence of the monodentate binding mode, others (incl. ECC with 0.8 scaling) predict an equilibrium between the two forms, even if the monodentate binding is always slightly favored. These findings are qualitatively consistent with earlier studies that suggested that $\mathrm{Ca}^{2+}$ slightly favored monodentate over bidentate binding, ${ }^{34,101}$ but that it was more prone to bidentate binding than $\mathrm{Mg}^{2+} .28,101$

To complement the picture provided by classical force field simulations — not always very conclusive especially for $\mathrm{Zn}^{2+}$ and $\mathrm{Ca}^{2+}$ - we performed DFT-based ab initio molecular dynamics simulations on small systems, using formate instead of acetate due to the large computational cost of such simulations (see Methods). We obtained for each cation the free energy profile (Figure 6) along the distance between the carboxylate carbon atom and the cation, in the region corresponding to the bidentate (short distances) to monodentate (larger distances) transformation. Due to the limited sampling allowed by these very expensive simulations, the error bar on the free energy 
profile is quite large, and difficult to estimate precisely, on the order of $4-5 \mathrm{~kJ} \mathrm{~mol}^{-1}$. However, the simulations are found robust to details of the simulation setup (in particular the dispersion correction, see Supplementary Information Fig. S5) and still bring very informative insight. Indeed, our AIMD simulations confirm that $\mathrm{Mg}^{2+}$-acetate ion pairs are only stable in a monodentate geometry (C-Mg distance of $3.1 \AA$ ), the bidentate ion pair being more than $25 \mathrm{~kJ}$ $\mathrm{mol}^{-1}$ higher in free energy. In contrast, both geometries appear possible and as probable (within the error bars) with $\mathrm{Ca}^{2+}$, again qualitatively confirming the picture from ECC force fields and previous AIMD simulations, ${ }^{11,102}$ but in contrast with the AMOEBA picture of exclusive monodentate pairing. For $\mathrm{Zn}^{2+}$, the AIMD simulations are in line with previous suggestions in the literature that the monodentate and bidentate binding modes are of similar stability, in contrast with what is predicted by all tested force fields, but in line with our comparison between measured and computed spectral shifts (see Results section).

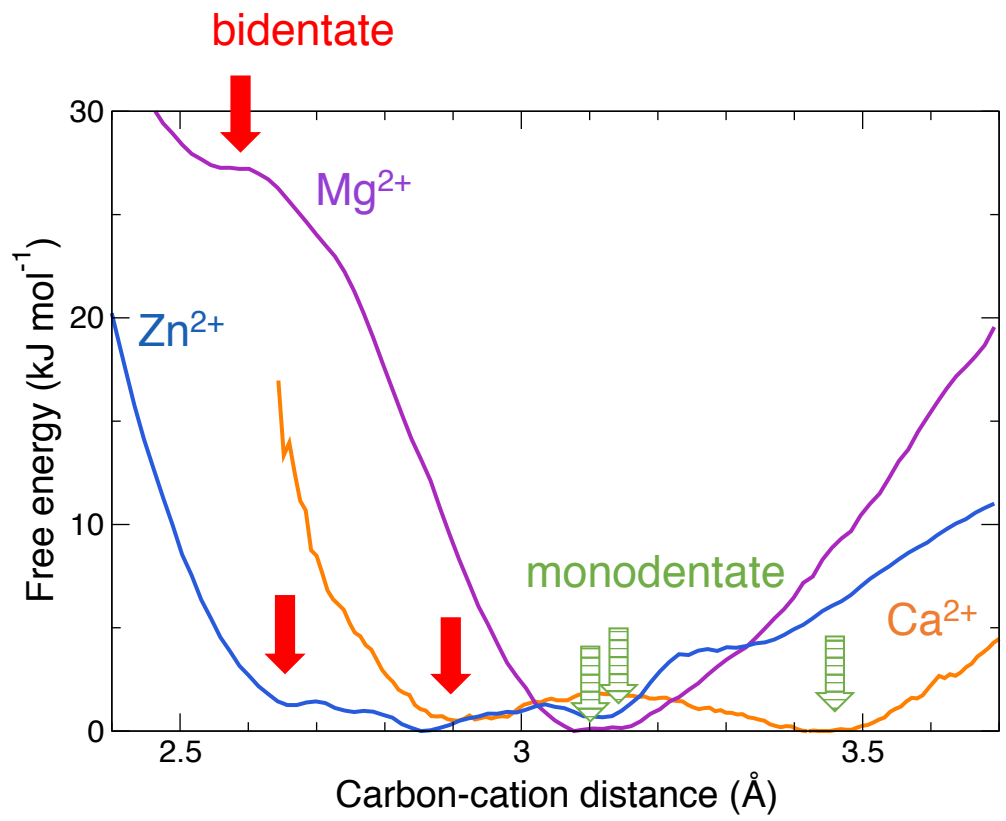

Figure 6. Free energy profiles along the carboxylate carbon-cation distance for calcium (orange), magnesium (purple) and zinc (blue) as obtained from DFT-based ab initio MD simulations. The location of the bidentate and monodentate ion pairs is indicated with red and dashed green arrows, respectively. No 
correction for the effective volume difference between the monodentate and bidentate structure has been done, because of the difficulty to estimate it properly given our limited sampling, but the largest expected correction, $-\mathrm{RT} \ln 2=-1.7 \mathrm{~kJ} / \mathrm{mol}$ in favor of the monodentate binding mode is well below the actual error bars of our simulation $(4-5 \mathrm{~kJ} / \mathrm{mol})$

\section{Conclusion}

In this study, we quantified binding of $\mathrm{Zn}^{2+}, \mathrm{Ca}^{2+}$, and $\mathrm{Mg}^{2+}$ to carboxylate anions using a combination of Raman-MCR spectroscopic measurements and both quantum and classical calculations, including molecular dynamics simulations with a force field that accounts for electronic polarization in a mean-field way via charge scaling. The Raman-MCR measurements yield self-consistent bound spectra and one-to-one binding constants demonstrating that $\mathrm{Zn}^{2+}$ binds about four times more strongly to acetate than either $\mathrm{Ca}^{2+}$ or $\mathrm{Mg}^{2+}$, in qualitative agreement with previous potentiometric measurements ${ }^{13,14}$ (and better quantitative agreement with more recently corrected potentiometric results ${ }^{15}$ for acetate binding to $\mathrm{Ca}^{2+}$ ). Results from the present molecular dynamics simulations agree semi-quantitatively with the Raman experimental data. Our simulations and quantum calculations further confirm that the Raman-MCR measurements are primarily sensitive to direct contact, as opposed to water-separated, ion pairing between the dications and the $\mathrm{COO}^{-}$group, and suggest that the experimentally detected bound species may include both monodentate and bidentate binding to $\mathrm{Zn}^{2+}$, but primarily monodentate binding to $\mathrm{Mg}^{2+}$ (over the experimental concentration range). Our results clearly demonstrate that the binding of acetate to these three cations does not scale with cation size, as both $\mathrm{Zn}^{2+}$ and $\mathrm{Mg}^{2+}$ are about $25 \%$ smaller than $\mathrm{Ca}^{2+} \cdot{ }^{103}$ The observed binding constants also do not scale with ion polarizability, as $\mathrm{Zn}^{2+}$ and $\mathrm{Ca}^{2+}$ are about twice as polarizable as $\mathrm{Mg}^{2+} \cdot{ }^{104}$ The more favorable binding of acetate to $\mathrm{Zn}^{2+}$ may perhaps be related to its higher electron binding affinity than either $\mathrm{Mg}^{2+}$ or $\mathrm{Ca}^{2+}$ (as 
inferred from the corresponding second ionization potentials), ${ }^{105}$ and perhaps also to the fact that the valence electrons of $\mathrm{Zn}^{2+}$ are in d-orbitals while those of $\mathrm{Mg}^{2+}$ and $\mathrm{Ca}^{2+}$ are in p-orbitals.

Our combined experimental and theoretical results demonstrate both the promise and outstanding challenges associated with quantifying non-covalent ion-paring interactions in aqueous solutions. The stronger acetate binding of $\mathrm{Zn}^{2+}$ than either $\mathrm{Mg}^{2+}$ or $\mathrm{Ca}^{2+}$ undoubtedly has biological implications to such processes as calcium signaling, stabilization of nucleic acids by magnesium, and zinc-mediated enzymatic activity. Our results also highlight the challenges associated with quantitatively linking experimental and theoretical ion-pairing results, including the need for improved cation force fields and improved procedures for resolving the range of ionpaired structures detected using different experimental methods.

\section{Acknowledgments}

DBA, DMdO, and SKZ were supported in part by the US National Science Foundation (CHE1763581). PJ thanks the Czech Science Foundation for support via an EXPRO grant no. 1926854X. EDD acknowledges support from the EMBO and Marie Curie Actions (fellowship ALTF 952-2015), and EDD and JH were supported in part by the "Initiative d'Excellence" program from the French State (Grants "DYNAMO", ANR-11-LABX-0011, and "CACSICE", ANR-11-EQPX0008). HMS and VP acknowledge support from the Czech Science Foundation (19-19561S). This work was performed with computer time allocated by the national supercomputing center IT4innovations in Ostrava (project OPEN-8-35), the European Regional Development Fund OP RDE (project ChemBioDrug no. CZ.02.1.01/0.0/0.0/16_019/0000729) computational resources, and the MetaCentrum data storage facilities. 


\section{References}

(1) Brini, M.; Ottolini, D.; Cali, T.; Carafoli, E. Calcium in Health and Disease; Sigel, A., Sigel, H., Sigel, R. K. O., Eds.; Springer Science+Business Media: Dordrec, 2013; Vol. 13.

(2) Clapham, D. E. Calcium Signaling. Cell 2007, 131, 1047-1058.

(3) Yamanaka, R.; Shindo, Y.; Oka, K. Magnesium Is a Key Player in Neuronal Maturation and Neuropathology. Int. J. Mol. Sci. 2019, 20, 3439.

(4) Romani, A. M. P. Magnesium in Health and Disease. In Interrelations between Essential Metal Ions and Human Diseases,; Sigel, A., Sigel, H., Sigel, R. K., Eds.; Springer Science+Business Media Dordrecht: Dordrecht, 2013; pp 49-79.

(5) de Baaij, J. H. F.; Hoenderop, J. G. J.; Bindels, R. J. M. Magnesium in Man: Implications for Health and Disease. Physiol. Rev. 2015, 95, 1-46.

(6) Coleman, J. E. Zinc Proteins : Enzymes, Storage Replication Proteins. Annu. Rev. Biochem. 1992, 61, 897-946.

(7) Frassinetti, S.; Bronzetti, G. L.; Caltavuturo, L.; Cini, M.; Croce, C. Della. The Role of Zinc in Life: A Review. J. Environ. Pathol. Toxicol. Oncol. 2006, 25, 597-610.

(8) Gifford, J. L.; Walsh, M. P.; Vogel, H. J. Structures and Metal-Ion-Binding Properties of the $\mathrm{Ca}^{2+}$-Binding Helix-Loop-Helix EF-Hand Motifs. Biochem. J. 2007, 405, 199-221.

(9) Klug, A.; Schwabe, J. W. Zinc Fingers. FASEB J. 1995, 9, 597-604.

(10) Hsiao, C.; Williams, L. D. A Recurrent Magnesium-Binding Motif Provides a Framework for the Ribosomal Peptidyl Transferase Center. Nucleic Acids Res. 2009, 37, 3134-3142.

(11) Martinek, T.; Duboué-Dijon, E.; Timr, Š.; Mason, P. E.; Baxová, K.; Fischer, H. E.; Schmidt, B.; Pluharová, E.; Jungwirth, P. Calcium Ions in Aqueous Solutions: Accurate Force Field Description Aided by Ab Initio Molecular Dynamics and Neutron Scattering. J. Chem. Phys. 2018, 148, 222813.

(12) Duboué-Dijon, E.; Mason, P. E.; Fischer, H. E.; Jungwirth, P. Hydration and Ion Pairing in Aqueous $\mathrm{Mg}^{2+}$ and $\mathrm{Zn}^{2+}$ Solutions: Force Field Description Aided by Neutron Scattering Experiments and Ab Initio Molecular Dynamics Simulations. J. Phys. Chem. B 2017, 122, 3296-3306.

(13) Martell, A. E.; Smith, R. M. Critical Stability Constants 3: Other Organic Ligands; 1977.

(14) Bunting, J.; Thong, K. M. Stability Constants for Some 1:1 Metal Carboxylate 
Complexes. Can. J. Chem. 1970, 48, 1654.

(15) Stumpff, F.; McGuigan, J. A. S. Measuring $\mathrm{Ca}^{2+}$ Binding to Short Chain Fatty Acids and Gluconate with a $\mathrm{Ca}^{2+}$ Electrode: Role of the Reference Electrode. Anal. Biochem. 2014, $459,46-52$.

(16) Bretti, C.; Majlesi, K.; De Stefano, C.; Sammartano, S. Thermodynamic Study on the Protonation and Complexation of GLDA with $\mathrm{Ca}^{2+}$ and $\mathrm{Mg}^{2+}$ at Different Ionic Strengths and Ionic Media at 298.15 K. J. Chem. Eng. Data 2016, 61, 1895-1903.

(17) McGuigan, J. A. S.; Kay, J. W.; Elder, H. Y. Ionised Concentrations in Calcium and Magnesium Buffers: Standards and Precise Measurement Are Mandatory. Prog. Biophys. Mol. Biol. 2016, 121, 195-211.

(18) Emara, M.; Farid, A.; Wasfi, A. Thermodynamics of Ionic Association in Aqueous Solutions of $\mathrm{Ca}$ and $\mathrm{Mg}$ Organic Salts Using Ion-Selective Electrode Technique. Electrochim. Acta 1980, 26, 1705-1708.

(19) Wallace, M.; Hicks, T.; Khimyak, Y. Z.; Angulo, J. Self-Correcting Method for the Measurement of Free Calcium and Magnesium Concentrations by 1H NMR. Anal. Chem. 2019, 91, 14442-14450.

(20) Tackett, J. E. FT-IR Characterization of Metal Acetates in Aqueous Solution. Appl. Spectrosc. 1989, 43, 483-489.

(21) Quilès, F.; Burneau, A. Infrared and Raman Spectra of Alkaline-Earth and Copper(II) / Acetates in Aqueous Solutions. Vib. Spectrosc. 1998, 16, 105-117.

(22) Rudolph, W. W.; Irmer, G. A Raman Spectroscopic Study of Aqueous La(CH3CO2)3 Solutions and $\mathrm{La}(\mathrm{CH} 3 \mathrm{CO} 2) 3 \cdot 1.5 \mathrm{H} 2 \mathrm{O}(\mathrm{Cr})$. J. Solution Chem. 2017, 46, 190-214.

(23) Tang, C. Y.; Huang, Z.; Allen, H. C. Binding of $\mathrm{Mg}^{2+}$ and $\mathrm{Ca}^{2+}$ to Palmitic Acid and Deprotonation of the $\mathrm{COOH}$ Headgroup Studied by Vibrational Sum Frequency Generation Spectroscopy. J. Phys. Chem. B 2010, 114, 17068-17076.

(24) Denton, J. K.; Kelleher, P. J.; Johnson, M. A.; Baer, M. D.; Kathmann, S. M.; Mundy, C. J.; Wellen Rudd, B. A.; Allen, H. C.; Choi, T. H.; Jordan, K. D. Molecular-Level Origin of the Carboxylate Head Group Response to Divalent Metal Ion Complexation at the AirWater Interface. Proc. Natl. Acad. Sci. 2019, 116, 14874-14880.

(25) Wahab, A.; Mahiuddin, S.; Hefter, G.; Kunz, W.; Minofar, B.; Jungwirth, P. Ultrasonic Velocities, Densities, Viscosities, Electrical Conductivities, Raman Spectra, and 
Molecular Dynamics Simulations of Aqueous Solutions of $\mathrm{Mg}(\mathrm{OAc}) 2$ and $\mathrm{Mg}(\mathrm{NO} 3) 2$ : Hofmeister Effects and Ion Pair Formation. J. Phys. Chem. B 2005, 109, 24108-24120.

(26) Mehandzhiyski, A. Y.; Riccardi, E.; van Erp, T. S.; Trinh, T. T.; Grimes, B. A. Ab Initio Molecular Dynamics Study on the Interactions between Carboxylate Ions and Metal Ions in Water. J. Phys. Chem. B 2015, 119, 10710-10719.

(27) Caminiti, R.; Cucca, P.; Monduzzi, M.; Saba, G.; Crisponi, G. Divalent Metal-Acetate Complexes in Concentrated Aqueous Solutions. An X-Ray Diffraction and NMR Spectroscopy Study. J. Chem. Phys. 1984, 81, 543-551.

(28) Dudev, T.; Lim, C. Principles Governing Mg, Ca, and Zn Binding and Selectivity in Proteins. Chem. Rev. 2003, 103, 773-787.

(29) Dudev, T.; Lim, C. Effect of Carboxylate-Binding Mode on Metal Binding / Selectivity. Acc Chem Res 2007, 40, 53-56.

(30) Ryde, U. Carboxylate Binding Modes in Zinc Proteins: A Theoretical Study. Biophys. J. 1999, 77, 2777-2787.

(31) Paterova, J.; Heyda, J.; Jungwirth, P.; Shaffer, C. J.; Revesz, A.; Zins, E. L.; Schroder, D. Microhydration of the Magnesium(II) Acetate Cation in the Gas Phase. J. Phys. Chem. A 2011, 115, 6813-6819.

(32) Semmler, J.; Irish, D. E.; Ozeki, T. Vibrational Spectral Studies of Solutions at Elevated Temperatures and Pressures. 12. Magnesium Acetate. Geochim. Cosmochim. Acta 1990, $54,947-954$.

(33) Wang, L. Y.; Zhang, Y. H.; Zhao, L. J. Raman Spectroscopic Studies on Single Supersaturated Droplets of Sodium and Magnesium Acetate. J. Phys. Chem. A 2005, 109, 609-614.

(34) Einspahr, H.; Bugg, C. E. The Geometry of Calcium Carboxylate Interactions in Crystalline Complexes. Acta Crystallogr. Sect. B Struct. Crystallogr. Cryst. Chem. 1981, 37, 1044-1052.

(35) Yang, M. M.; Crerar, D. A.; Irish, D. E. A Raman Spectroscopic Study of Lead and Zinc Acetate Complexes in Hydrothermal Solutions. Geochim. Cosmochim. Acta 1989, 53, 319-326.

(36) Katz, A. K.; Glusker, J. P.; Markham, G. D.; Bock, C. W. Deprotonation of Water in the Presence of Carboxylate and Magnesium Ions. J. Phys. Chem. B 1998, 102, 6342-6350. 
(37) Dudev, T.; Lim, C. Monodentate versus Bidentate Carboxylate Binding in Magnesium and Calcium Proteins: What Are the Basic Principles? J. Phys. Chem. B 2004, 108, 45464557.

(38) Humphrey, W., Dalke, A. and Schulten, K. VMD - Visual Molecular Dynamics. J. Molec. Graph. 1996, 14, 33-38.

(39) Davis, J. G.; Gierszal, K. P.; Wang, P.; Ben-Amotz, D. Water Structural Transformation at Molecular Hydrophobic Interfaces. Nature 2012, 491, 582-585.

(40) Gierszal, K. P.; Davis, J. G.; Hands, M. D.; Wilcox, D. S.; Slipchenko, L. V.; Ben-Amotz, D. П-Hydrogen Bonding in Liquid Water. J. Phys. Chem. Lett. 2011, 2, 2930-2933.

(41) Lawton, W. H.; Sylvestre, E. A. Self Modeling Curve Resolution. Technometrics 1971, 13, 617-633.

(42) Perera, P. N.; Browder, B.; Ben-Amotz, D. Perturbations of Water by Alkali Halide Ions Measured Using Multivariate Raman Curve Resolution. J. Phys. Chem. B 2009, 113, 1805-1809.

(43) Van der Spoel, D.; Lindahl, E.; Hess, B.; Groenhof, G.; Mark, A. E.; Berendsen, H. J. C. GROMACS: Fast, Flexible, and Free. J. Comput. Chem. 2005, 26, 1701-1718.

(44) Parrinello, M.; Rahman, A. Polymorphic Transitions in Single Crystals: A New Molecular Dynamics Method. J. Appl. Phys. 1981, 52, 7182-7190.

(45) Bussi, G.; Donadio, D.; Parrinello, M. Canonical Sampling through Velocity Rescaling. J. Chem. Phys. 2007, 126, 014101.

(46) Darden, T.; York, D.; Pedersen, L. Particle Mesh Ewald: An Nlog(N) Method for Ewald Sums in Large Systems. J. Chem. Phys. 1993, 98, 10089-10092.

(47) Hess, B.; Bekker, H.; Berendsen, H. J. C.; Fraaije, J. G. E. M. LINCS: A Linear Constraint Solver for Molecular Simulations. J. Comput. Chem. 1997, 18, 1463-1472.

(48) Miyamoto, S.; Kollman, P. A. SETTLE: An Analytical Version of the SHAKE and RATTLE Algorithm for Rigid Water Models. J. Comput. Chem. 1992, 13, 952-962.

(49) Berendsen, H. J. C.; Grigera, J. R.; Straatsma, T. P. The Missing Term in Effective Pair Potentials. J. Phys. Chem. 1987, 91, 6269-6271.

(50) Joung, I. S.; Cheatham, T. E. Determination of Alkali and Halide Monovalent Ion Parameters for Use in Explicitly Solvated Biomolecular Simulations. J. Phys. Chem. B 2008, 112, 9020-9041. 
(51) Li, P.; Roberts, B. P.; Chakravorty, D. K.; Merz, K. M. Rational Design of Particle Mesh Ewald Compatible Lennard-Jones Parameters for +2 Metal Cations in Explicit Solvent.

J.Chem. Theory Comput. 2013, 9, 2733-2748.

(52) Mamatkulov, S.; Fyta, M.; Netz, R. R. Force Fields for Divalent Cations Based on SingleIon and Ion-Pair Properties. J. Chem. Phys. 2013, 138, 024505.

(53) Callahan, K. M.; Casillas-Ituarte, N. N.; Roeselová, M.; Allen, H. C.; Tobias, D. J. Solvation of Magnesium Dication: Molecular Dynamics Simulation and Vibrational Spectroscopic Study of Magnesium Chloride in Aqueous Solutions. J. Phys. Chem. A 2010, 114, 5141-5148.

(54) Stote, R. H.; Karplus, M. Zinc Binding in Proteins and Solution: A Simple but Accurate Nonbonded Representation. Proteins 1995, 23, 12-31.

(55) Leontyev, I. V.; Stuchebrukhov, A. A. Electronic Continuum Model for Molecular Dynamics Simulations. J. Chem. Phys. 2009, 130, 085102.

(56) Leontyev, I.; Stuchebrukhov, A. Accounting for Electronic Polarization in NonPolarizable Force Fields. Phys. Chem. Chem. Phys. 2011, 13, 2613-2626.

(57) Kirby, B. J.; Jungwirth, P. Charge Scaling Manifesto: A Way of Reconciling the Inherently Macroscopic and Microscopic Natures of Molecular Simulations. J. Phys. Chem. Lett. 2019, 10, 7531-7536.

(58) Kohagen, M.; Mason, P. E.; Jungwirth, P. Accounting for Electronic Polarization Effects in Aqueous Sodium Chloride via Molecular Dynamics Aided by Neutron Scattering. $J$. Phys. Chem. B 2016, 120, 1454-1460.

(59) Kohagen, M.; Mason, P. E.; Jungwirth, P. Accurate Description of Calcium Solvation in Concentrated Aqueous Solutions. J. Phys. Chem. B 2014, 118, 7902-7909.

(60) Duboué-Dijon, E. Supporting Data Files for "Binding of Biologically Relevant Divalent Cations to Aqueous Carboxylates: Molecular Simulations Guided by Raman Spectroscopy." 2020, doi: 10.5281/zenodo.3827165.

(61) Jorgensen, W. L.; Buckner, J. K.; Boudon, S.; Tirado-Rives, J. Efficient Computation of Absolute Free Energies of Binding by Computer Simulations. Application to the Methane Dimer in Water. J. Chem. Phys. 1988, 89, 3742-3746.

(62) Hermans, J.; Shankar, S. The Free Energy of Xenon Binding to Myoglobin from Molecular Dynamics Simulation. Isr. J. Chem. 1986, 27, 225-227. 
(63) Boresch, S.; Tettinger, F.; Leitgeb, M.; Karplus, M. Absolute Binding Free Energies: A Quantitative Approach for Their Calculation. J. Phys. Chem. B 2003, 107, 9535-9551.

(64) Roux, B.; Nina, M.; Pomès, R.; Smith, J. C. Thermodynamic Stability of Water Molecules in the Bacteriorhodopsin Proton Channel: A Molecular Dynamics Free Energy Perturbation Study. Biophys. J. 1996, 71, 670-681.

(65) Bennett, C. H. Efficient Estimation of Free Energy Differences from Monte Carlo Data. J. Comput. Phys. 1976, 22, 245-268.

(66) de Leeuw, S. W.; Perram, J. W.; Smith, E. R. Simulation of Electrostatic Systems in Periodic Boundary Conditions. I. Lattice Sums and Dielectric Constants. Proc. R. Soc. A Math. Phys. Eng. Sci. 1980, 373, 27-56.

(67) Hummer, G.; Pratt, L. R.; Angel E. Garcia. On the Free Energy of Ionic Hydration. $J$. Chem. Phys. 1956, 24, 357-359.

(68) Simonson, T.; Roux, B. Concepts and Protocols for Electrostatic Free Energies. Mol. Simul. 2016, 42, 1090-1101.

(69) Kumar, M.; Simonson, T.; Ohanessian, G.; Clavaguéra, C. Structure and Thermodynamics of Mg:Phosphate Interactions in Water: A Simulation Study. ChemPhysChem 2015, 16, $658-665$.

(70) Mobley, D. L.; Chodera, J. D.; Dill, K. A. On the Use of Orientational Restraints and Symmetry Corrections in Alchemical Free Energy Calculations. J. Chem. Phys. 2006, 125,084902 .

(71) Wang, J.; Deng, Y.; Roux, B. Absolute Binding Free Energy Calculations Using Molecular Dynamics Simulations with Restraining Potentials. Biophys. J. 2006, 91, 27982814.

(72) Vandevondele, J.; Krack, M.; Mohamed, F.; Parrinello, M.; Chassaing, T.; Hutter, J. QUICKSTEP: Fast and Accurate Density Functional Calculations Using a Mixed Gaussian and Plane Waves Approach. Comput. Phys. Comm. 2005, 167, 103-128.

(73) Becke, A. D. Density-Functional Exchange-Energy Approximation with Correct Asymptotic Behavior. Phys. Rev. A 1988, 38, 3098-3100.

(74) Lee, C.; Yang, W.; Parr, R. G. Development of the Colle-Salvetti Correlation-Energy Formula into a Functional of the Electron Density. Phys. Rev. B 1988, 37, 785-789.

(75) Grimme, S.; Antony, J.; Ehrlich, S.; Krieg, H. A Consistent and Accurate Ab Initio 
Parametrization of Density Functional Dispersion Correction (DFT-D) for the 94

Elements H-Pu. J. Chem. Phys. 2010, 132, 154104.

(76) Smith, D. G. A.; Burns, L. A.; Patkowski, K.; Sherrill, C. D. Revised Damping Parameters for the D3 Dispersion Correction to Density Functional Theory. J. Phys. Chem. Lett. 2016, 7, 2197-2203.

(77) Becke, A. D.; Johnson, E. R. A Density-Functional Model of the Dispersion Interaction. $J$. Chem. Phys. 2005, 123, 154101.

(78) Grimme, S. Semiempirical GGA-Type Density Functional Constructed with a LongRange Dispersion Correction. J. Comput. Chem. 2006, 27, 1787-1799.

(79) VandeVondele, J.; Hutter, J. Gaussian Basis Sets for Accurate Calculations on Molecular Systems in Gas and Condensed Phases. J. Chem. Phys. 2007, 127, 114105.

(80) Goedecker, S.; Teter, M.; Hutter, J. Separable Dual-Space Gaussian Pseudopotentials. Phys. Rev. B 1996, 54, 1703-1710.

(81) Kumar, S.; Rosenberg, J. M.; Bouzida, D.; Swendsen, R. H.; Kollman, P. A. Multidimensional Free-Energy Calculations Using the Weighted Histogram Analysis Method. J. Comput. Chem. 1995, 16, 1339-1350.

(82) Grossfield, A. An Implementation of WHAM: The Weighted Histogram Analysis Method. Analysis 2004, 1, 1-13.

(83) Frisch, M. J.; Trucks, G. W.; Schlegel, H. B.; Scuseria, G. E.; Robb, M. A.; Cheeseman, J. R.; Scalmani, G.; Barone, V.; Petersson, G. A.; Nakatsuji, H.; et al. Gaussian 16 Revision C.01. 2016.

(84) Jorgensen, W. L.; Chandrasekhar, J.; Madura, J. D.; Impey, R. W.; Klein, M. L. Comparison of Simple Potential Functions for Simulating Liquid Water. J. Chem. Phys. 1983, 79, 926 .

(85) Dapprich, S.; Komáromi, I.; Byun, K. S.; Morokuma, K.; Frisch, M. J. A New ONIOM Implementation in Gaussian98. Part I. The Calculation of Energies, Gradients, Vibrational Frequencies and Electric Field Derivatives. J. Mol. Struct. 1999, 461-462, 1-21.

(86) Ruud, K.; Helgaker, T.; Bouř, P. Gauge-Origin Independent Density-Functional Theory Calculations of Vibrational Raman Optical Activity. J. Phys. Chem. A 2002, 106, 74487455 .

(87) Palivec, V.; Kopecky Jr., V.; Jungwirth, P.; Bour, P.; Kaminsky, J.; Martinez-Seara, H. 
Simulation of Raman and Raman Optical Activity of Saccharides in Solution. PCCP 2020, DOI: $10.1039 / \mathrm{c} 9 \mathrm{cp} 05682 \mathrm{c}$.

(88) Davis, J. G.; Zukowski, S. R.; Rankin, B. M.; Ben-Amotz, D. Influence of a Neighboring Charged Group on Hydrophobic Hydration Shell Structure. J. Phys. Chem. B 2015, 119, 9417-9422.

(89) Quilès, F.; Burneau, A.; Gross, N. Vibrational Spectroscopic Study of the Complexation of Mercury(II) by Substituted Acetates in Aqueous Solutions. Appl. Spectrosc. 1999, 53, 1061-1070.

(90) Uejio, J. S.; Schwartz, C. P.; Duffin, A. M.; Drisdell, W. S.; Cohen, R. C.; Saykally, R. J. Characterization of Selective Binding of Alkali Cations with Carboxylate by X-Ray Absorption Spectroscopy of Liquid Microjets. Proc. Natl. Acad. Sci. 2008, 105, 68096812.

(91) Duboué-Dijon, E.; Delcroix, P.; Martinez-Seara, H.; Hladilkova, J.; Coufal, P.; Krizek, T.; Jungwirth, P. Binding of Divalent Cations to Insulin: Capillary Electrophoresis and Molecular Simulations. J. Phys. Chem. B 2018, 122, 5640-5648.

(92) Ponder, J. W.; Wu, C.; Pande, V. S.; Chodera, J. D.; Schnieders, M. J.; Haque, I.; Mobley, D. L.; Lambrecht, D. S.; Distasio, R. a; Head-gordon, M.; et al. Current Status of the AMOEBA Polarizable Force Field. J. Phys. Chem. B 2010, 114, 2549-2564.

(93) Villa, F.; Mackerell, A. D.; Roux, B.; Simonson, T. Classical Drude Polarizable Force Field Model for Methyl Phosphate and Its Interactions with $\mathrm{Mg}^{2+}$. J. Phys. Chem. A 2018, $122,6147-6155$.

(94) Lemkul, J. A.; Huang, J.; Roux, B.; Mackerell, A. D. An Empirical Polarizable Force Field Based on the Classical Drude Oscillator Model: Development History and Recent Applications. Chem. Rev. 2016, 116, 4983-5013.

(95) Simonson, T.; Satpati, P. Simulating GTP:Mg and GDP:Mg with a Simple Force Field: A Structural and Thermodynamic Analysis. J. Comput. Chem. 2013, 34, 836-846.

(96) Davies, C. W. The Extent of Dissociation of Salts in Water. Part VIII. An Equation for the Mean Ionic Activity Coefficient of an Electrolyte in Water, and a Revision of the Dissociation Constants of Some Sulphates. J. Chem. Soc. 1938, 2093-2098.

(97) Sun, M. S.; Harriss, D. K.; Magnuson, R. Activity Corrections for Ionic Equilibria in Aqueous Solutions. Can. J. Chem. 1980, 58, 1253-1257. 
(98) Kann, Z. R.; Skinner, J. L. A Scaled-Ionic-Charge Simulation Model That Reproduces Enhanced and Suppressed Water Diffusion in Aqueous Salt Solutions. J. Chem. Phys. 2014, 141, 104507.

(99) Zeron, I. M.; Abascal, J. L. F.; Vega, C. A Force Field of Li , Na , K , Mg , Ca , in Aqueous Solution Based on the TIP4P / 2005 Water Model and Scaled Charges for the Ions in Aqueous Solution Based on the TIP4P / 2005 Water Model and Scaled Charges for the Ions. J. Chem. Phys. 2019, 151, 134504.

(100) Caminiti, R.; Licheri, G.; Piccaluga, G.; Pinna, G. X-Ray Diffraction Study of MgCl2 Aqueous Solutions. J. Appl. Cryst. 1979, 12, 34-38.

(101) Katz, A. K.; Glusker, J. P.; Beebe, S. A.; Bock, C. W. Calcium Ion Coordination: A Comparison with That of Beryllium, Magnesium, and Zinc. J. Am. Chem. Soc. 1996, 118, $5752-5763$.

(102) Daily, M. D.; Baer, M. D.; Mundy, C. J. Divalent Ion Parameterization Strongly Affects Conformation and Interactions of an Anionic Biomimetic Polymer. J. Phys. Chem. B 2016, 120, 2198-2208.

(103) Shannon, R. D. Revised Effective Ionic Radii and Systematic Studies of Interatomie Distances in Halides and Chaleogenides. Acta Cryst. A 1976, 32, 751-767.

(104) Shannon, R. D.; Fischer, R. X. Empirical Electronic Polarizabilities of Ions for the Prediction and Interpretation of Refractive Indices: Oxides and Oxysalts. Am. Mineral. 2016, 101, 2288-2300.

(105) Linstrom, P. J.; Mallard, W. G. NIST Chemistry WebBook, NIST Standard Reference Database Number 69; Linstrom, P. J., Mallard, W. G., Eds.; National Institute of Standards and Technology, Gaithersburg MD, 20899. 